\title{
BMJ Open Overdiagnosis across medical disciplines: a scoping review
}

\author{
Kevin Jenniskens, ${ }^{1}$ Joris A H de Groot, ${ }^{1}$ Johannes B Reitsma, ${ }^{1,2}$ \\ Karel G M Moons, ${ }^{1,2}$ Lotty Hooft, ${ }^{1,2}$ Christiana A Naaktgeboren ${ }^{1}$
}

To cite: Jenniskens $\mathrm{K}$, de Groot JAH, Reitsma JB, et al. Overdiagnosis across medical disciplines: a scoping review. BMJ Open 2017;7:e018448. doi:10.1136/ bmjopen-2017-018448

- Prepublication history and additional material for this paper are available online. To view these files, please visit the journal online (http://dx.doi. org/10.1136/bmjopen-2017018448).

Received 29 June 2017 Revised 3 November 2017 Accepted 8 November 2017

CrossMark

${ }^{1}$ Julius Center for Health Sciences and Primary Care, University Medical Center Utrecht, Utrecht, The Netherlands

${ }^{2}$ Dutch Cochrane Centre, University Medical Center Utrecht, Utrecht, The Netherlands

Correspondence to Kevin Jenniskens;

k.jenniskens@umcutrecht.nl

\section{ABSTRACT}

Objective To provide insight into how and in what clinical fields overdiagnosis is studied and give directions for further applied and methodological research.

Design Scoping review.

Data sources Medline up to August 2017.

Study selection All English studies on humans, in which overdiagnosis was discussed as a dominant theme.

Data extraction Studies were assessed on clinical field, study aim (ie, methodological or non-methodological), article type (eg, primary study, review), the type and role of diagnostic test(s) studied and the context in which these studies discussed overdiagnosis.

Results From 4896 studies, 1851 were included for analysis. Half of all studies on overdiagnosis were performed in the field of oncology (50\%). Other prevalent clinical fields included mental disorders, infectious diseases and cardiovascular diseases accounting for 9\%, $8 \%$ and $6 \%$ of studies, respectively. Overdiagnosis was addressed from a methodological perspective in $20 \%$ of studies. Primary studies were the most common article type (58\%). The type of diagnostic tests most commonly studied were imaging tests (32\%), although these were predominantly seen in oncology and cardiovascular disease (84\%). Diagnostic tests were studied in a screening setting in $43 \%$ of all studies, but as high as $75 \%$ of all oncological studies. The context in which studies addressed overdiagnosis related most frequently to its estimation, accounting for $53 \%$. Methodology on overdiagnosis estimation and definition provided a source for extensive discussion. Other contexts of discussion included definition of disease, overdiagnosis communication, trends in increasing disease prevalence, drivers and consequences of overdiagnosis, incidental findings and genomics.

Conclusions Overdiagnosis is discussed across virtually all clinical fields and in different contexts. The variability in characteristics between studies and lack of consensus on overdiagnosis definition indicate the need for a uniform typology to improve coherence and comparability of studies on overdiagnosis.

\section{INTRODUCTION}

Overmedicalisation is the broad overarching term describing the use of 'too much medicine'. It encompasses various concepts such as disease mongering, misdiagnosis, overutilisation, overdetection and overtreatment. Initiatives relating to these concepts have

\section{Strengths and limitations of this study}

- First complete overview of overdiagnosis across medical disciplines.

- Identification of the dominant clinical fields in which overdiagnosis is being studied, what characteristics these papers have and in what context it is being studied.

- Not a fully comprehensive systematic review, due to widespread variation in terminology and concepts used related to overdiagnosis.

- Studies on incidental findings were likely missed due to usage of different terminology to describe overdiagnosis.

begun to flourish on a global scale under the 'Choosing Wisely' initiative and in national programme such as Slow Medicine (Italy, the Netherlands and Brazil), Quaternary Prevention (Belgium) and Do not do (UK). ${ }^{2}{ }^{3} \mathrm{~A}$ subcategory of the aforementioned concepts is overdiagnosis. This has become an even more popular term especially over the last two decades. ${ }^{4-9}$ Furthermore, an annual conference going by the name of 'Preventing Overdiagnosis', dedicated to issues surrounding this concept, has been gaining popularity ever since its start in 2013, demonstrating a growing interest in the topic. ${ }^{10}$ In this scoping review, we will focus specifically on overdiagnosis.

Defining overdiagnosis is challenging and diverse definitions exist. ${ }^{11} 12$ In a narrow sense, overdiagnosis describes individuals receiving a diagnosis with a condition that would never have become symptomatic before the end of the individual's life. ${ }^{57}$ However, overdiagnosis has also been described as giving a diagnosis that would not yield a net benefit. ${ }^{1}$ These definitions are not similar and thus may lead to different interpretations of (the extent of) overdiagnosis. Consequently, the mechanisms leading to overdiagnosis may also differ. Labelling an individual with a blood pressure over a certain threshold as hypertensive, and thus 'diseased', is conceptually different from not knowing whether one should diagnose an 


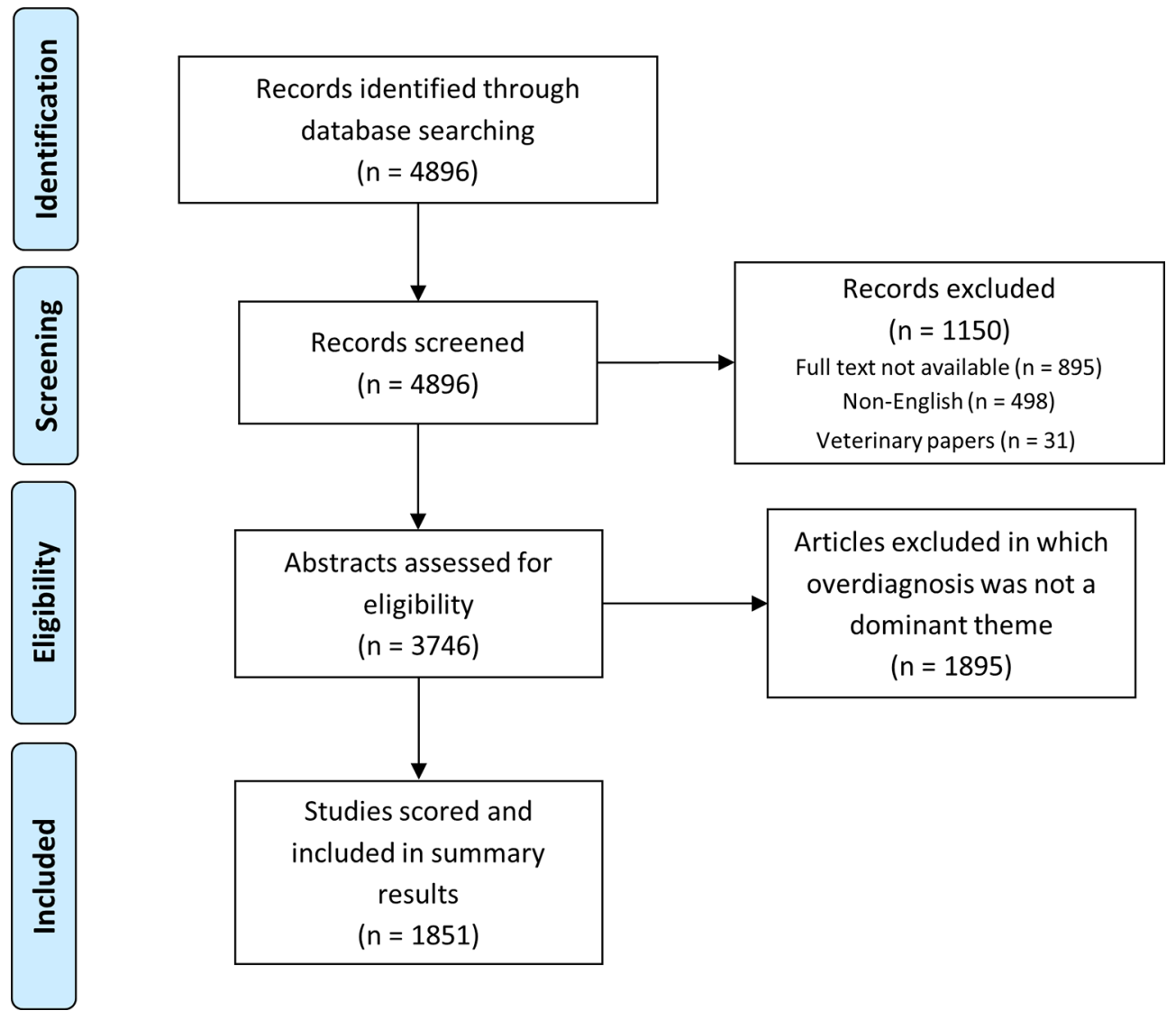

Figure 1 Flow diagram of article selection for further review and scoring.

individual with a very small potentially malignant growth as having cancer. Providing definitions in combination with mechanisms of overdiagnosis for a typology is challenging and source of extensive discussion. ${ }^{13-17}$

The range of overdiagnosis drivers is also extensive. It, among others, includes technological developments that detect smaller abnormalities than ever before which might not become clinically manifest. Furthermore, the use of large-scale screening programme, inappropriate application of diagnostic criteria, legal incentives, cultural believes (ie, that we should do everything in our power to find and treat disease) and commercial or professional interests have driven overdiagnosis. ${ }^{6} 18-20$

Consequences of overdiagnosis may be serious and can be subdivided in negative effects on patient health and additional costs within the healthcare system. ${ }^{21}$ Health effects include impaired quality of life and early loss of life due to side effects or complications of unnecessary subsequent testing or treatment. Incorrectly labelling of individuals as patients may also lead to stigmatisation, impacting psychological well-being and indirectly exert social effects through eligibility for health benefits. In monetary terms, overdiagnosis can result in unwarranted usage of (follow-up) tests, treatment and healthcare facilities and services.

Despite the increasing number of publications on overdiagnosis, ranging from discussions on overdiagnosis definition to estimating its impact, a scoping analysis on overdiagnosis is still lacking. In the present study, we provide an overview of research that has been performed across medical disciplines surrounding the topic of overdiagnosis. We will give insight into how and in what clinical fields overdiagnosis is studied and provide directions for further applied and methodological research to investigate the mechanisms and impact of overdiagnosis and to generate directions for reducing or preventing overdiagnosis.

\section{METHODS}

PubMed was searched on August 2017 for published articles using keywords related to overdiagnosis, overdetection, overscreening, insignificant disease, overtesting, overmedicalisation, pseudodisease, inconsequential disease and quaternary prevention by using the following query: overdiagnos*[tw] OR over diagnos*[tw] OR overdetect*[tw] OR over detect*[tw] OR insignificant disease' $[\mathrm{tw}]$ OR overscreen*[tw] OR over screen*[tw] OR overtest*[tw] OR over test*[tw] OR overmedical*[tw] OR over medical*[tw] OR 'pseudodisease' $[\mathrm{tw}]$ OR 'pseudo disease' $[\mathrm{tw}]$ OR 'inconsequential disease' $[\mathrm{tw}]$ OR 'Quaternary prevention' [tw].

These terms were chosen as they were believed to capture most concepts related to overdiagnosis, generating a representative set of articles. All English articles on humans where full text was available were included. 
Table 1 Characteristics of papers in which overdiagnosis was a dominant theme, with the results shown for the total number of articles, the four largest clinical fields, all other remaining clinical fields and studies not addressing a specific clinical field

\begin{tabular}{|c|c|c|c|c|c|c|c|}
\hline & $\begin{array}{l}\text { Total } \\
(n=1851) \\
(\%)\end{array}$ & $\begin{array}{l}\text { Oncological } \\
\text { disorders } \\
(\mathrm{n}=920) \\
(\%)\end{array}$ & $\begin{array}{l}\text { Mental } \\
\text { disorders } \\
(n=171) \\
(\%)\end{array}$ & $\begin{array}{l}\text { Infectious } \\
\text { diseases } \\
(n=143) \\
(\%)\end{array}$ & $\begin{array}{l}\text { Cardiovascular } \\
\text { disorders } \\
(n=105) \\
(\%)\end{array}$ & $\begin{array}{l}\text { Other clinical } \\
\text { fields } \\
(\mathrm{n}=390) \\
(\%)\end{array}$ & $\begin{array}{l}\text { No specific } \\
\text { clinical field } \\
(n=122) \\
(\%)\end{array}$ \\
\hline \multicolumn{8}{|l|}{ Study aim } \\
\hline Methodological & 20 & 30 & 11 & 4 & 10 & 4 & 34 \\
\hline Non-methodological & 80 & 70 & 89 & 96 & 90 & 96 & 66 \\
\hline \multicolumn{8}{|l|}{ Article type } \\
\hline Primary study & 58 & 55 & 53 & 85 & 61 & 69 & 27 \\
\hline Narrative review & 24 & 22 & 32 & 9 & 24 & 22 & 52 \\
\hline Systematic review & 9 & 12 & 8 & 1 & 10 & 5 & 11 \\
\hline Commentary & 9 & 11 & 8 & 6 & 6 & 4 & 10 \\
\hline \multicolumn{8}{|l|}{ Diagnostic test } \\
\hline Imaging & 32 & 48 & 3 & 4 & 47 & 19 & 7 \\
\hline Medical examination & 17 & 3 & 58 & 26 & 26 & 30 & 4 \\
\hline Biomarker & 15 & 16 & 3 & 29 & 10 & 16 & 3 \\
\hline Histology & 13 & 17 & 0 & 21 & 2 & 11 & 2 \\
\hline Prediction model & 3 & 4 & 1 & 2 & 3 & 4 & 1 \\
\hline Various & 21 & 13 & 35 & 18 & 12 & 20 & 84 \\
\hline \multicolumn{8}{|l|}{ Screening } \\
\hline Yes & 43 & 75 & 5 & 10 & 15 & 10 & 20 \\
\hline No & 57 & 25 & 95 & 90 & 85 & 90 & 80 \\
\hline \multicolumn{8}{|l|}{ Overdiagnosis context } \\
\hline $\begin{array}{l}\text { Overdiagnosis } \\
\text { estimation }\end{array}$ & 53 & 57 & 22 & 63 & 65 & 60 & 16 \\
\hline Disease definition & 15 & 8 & 46 & 13 & 14 & 22 & 8 \\
\hline $\begin{array}{l}\text { Overdiagnosis } \\
\text { communication }\end{array}$ & 3 & 5 & 2 & 0.7 & 0 & 0.8 & 3 \\
\hline Incidental findings & 0.8 & 0.8 & 0 & 0 & 1 & 1 & 2 \\
\hline Genomics & 0.4 & 0.3 & 0 & 0 & 1 & 0 & 3 \\
\hline Other ${ }^{\star}$ & 28 & 29 & 30 & 24 & 19 & 16 & 67 \\
\hline
\end{tabular}

*Subcategories in this category include: overdiagnosis definition, drivers and consequences of overdiagnosis and trend studies suggesting overdiagnosis.

Articles in which overdiagnosis was a dominant theme were included. Overdiagnosis was considered a dominant theme when a paper clearly addressed overdiagnosis as an issue being investigated or discussed. For example, a study on the adoption of a new threshold guideline for prostate-specific antigen screening for prostate cancer was considered to have a dominant overdiagnosis theme. In contrast, a study that used overdiagnosis as a buzzword and merely suggested in the discussion that overdiagnosis might possibly play a role or have occurred, was excluded. Studies with overdiagnosis as a dominant theme were included regardless of which definition of overdiagnosis the authors adopted.

The titles and abstracts of the included studies were then screened. Included studies were assessed using (a list of) prespecified criteria. These criteria were established by screening the first 200 studies of the search query. They included clinical field, study aim, article type, type of diagnostic test, whether this was a screening test and the context in which overdiagnosis was discussed. These criteria are described below (see further details in the online supplementary file 1). Articles were assessed based solely on title and abstract. If an abstract was unavailable (eg, opinion pieces), the full text was scanned.

\section{Clinical field}

The clinical field to which the study belonged was determined using the ICD-10 classification. When a study addressed more than one clinical field or did not address overdiagnosis within a specific clinical field, but discussed 
overdiagnosis on a more general level, they were included in the separate category 'no specific clinical field'.

\section{Study aim}

Two study aims were distinguished: (1) studies focusing on how overdiagnosis should be studied. These are studies with a methodological aim. Examples are studies looking into how overdiagnosis estimations are affected by the methods used, or studies providing a framework for the definition of overdiagnosis. Simulation studies using mathematical models for estimating the extent of overdiagnosis were also classified as methodological studies. Studies not addressing the aforementioned concepts, but rather provide, for example, a qualitative overview of the (possible) impact of overdiagnosis in a certain field, or calculate overdiagnosis estimates from empirical data, were considered to have (2) a non-methodological aim.

\section{Article types}

Studies were classified using four article types: primary studies, narrative reviews, systematic reviews or commentaries. Primary studies used data collected from trials, observational studies or generated using simulation models. Narrative reviews described a broad oversight on overdiagnosis. These included editorials, opinion pieces, interviews and overviews. Systematic reviews stated a specific hypothesis and tested this using a systematic approach to gather existing literature. If a systematic approach was lacking, these studies were scored as narrative reviews. Studies were considered commentaries when they, replied to previously published papers.

\section{Types of diagnostic test}

Diagnostic tests were categorised into six types: imaging, medical examination, biomarker, histology, prediction model or various. Whenever a study looked into a combination of two tests, both types were scored. For example, an image-guided biopsy would be scored as both an imaging and histologic diagnostic test. If three or more diagnostic tests were addressed within a study, or overdiagnosis was addressed in a general context without any diagnostic test in particular, this was scored under 'various tests'.

\section{Screening}

When studies focused on a test used for screening groups of asymptomatic individuals, this was scored as a screening study. Studies that did not explicitly state that the diagnostic test was studied in the context of screening, were scored as a non-screening.

\section{Overdiagnosis context}

To assess the context in which studies discussed overdiagnosis five categories were defined: estimating extent of overdiagnosis, disease definition, overdiagnosis communication, incidental findings and genomics. The first category, estimating extent of overdiagnosis, relates to all articles giving a quantified estimate of overdiagnosis. Disease definition revolves around the setting of thresholds to define the absence or presence of a disease or to distinguish between two subcategories of a certain disease (eg, progressive and non-progressive forms). Overdiagnosis communication relates to studies aimed at assessing and improving the understanding of overdiagnosis in the general public and improving overdiagnosis dissemination by the healthcare professionals. Studies addressing abnormalities found of an unrelated condition during either diagnostic testing or surgery were scored as studies on incidental findings. Spurious findings on genome wide screening tests were scored in the overdiagnosis context of genomics.

\section{RESULTS}

The PubMed search resulted in a total number of 4896 studies identified. After application of the inclusion criteria, 3746 studies were assessed for eligibility on title and abstract. Studies in which overdiagnosis was a dominant theme yielded 1851 studies (figure 1). Table 1 provides a summarised view of the characteristics of the total number of studies, the four largest clinical fields, all other remaining clinical fields and studies not related to a specific clinical field.

\section{Clinical field}

Papers on overdiagnosis were found in all clinical fields, but were mainly published within oncology $(50 \%)$, in which breast $(34 \%)$, prostate $(24 \%)$ and lung cancer (14\%) ranked as most prevalently studied. Other clinical fields addressing overdiagnosis included mental disorders $(9 \%)$, infectious diseases $(8 \%)$ and cardiovascular disease $(6 \%)$. Within these fields, studies were predominantly looking into bipolar disorder, malaria and pulmonary embolism, respectively. ${ }^{22-27}$

\section{Study aim}

Studies addressing methodological issues consisted of 20\%. The majority of these studies were performed within the field of oncology. However, non-methodological studies were the most common study aim used across all clinical fields, accounting for $80 \%$ of the total number of articles. These notably included studies using empirical data to assess the occurrence or estimate overdiagnosis for a specific disease.

\section{Article type}

Primary studies $(58 \%)$ were the most common article type discussing overdiagnosis. Of all included studies narrative, systematic reviews and commentaries represented 24\%, 9\% and 9\%, respectively. From all studies that addressed a specific clinical field, the proportion of systematic reviews and commentaries was relatively high within oncology.

\section{Type of diagnostic test}

Imaging was the most often encountered diagnostic test, accounting for $32 \%$ of all studies. Biomarkers (15\%), histology (13\%) and medical examination (17\%) were approximately equally often found. Prediction models 
Table 2 Descriptions and examples of context of overdiagnosis discussion

\begin{tabular}{|c|c|c|}
\hline $\begin{array}{l}\text { Overdiagnosis } \\
\text { context }\end{array}$ & Description & Example \\
\hline $\begin{array}{l}\text { Overdiagnosis } \\
\text { estimation }\end{array}$ & Providing a quantitative estimate of overdiagnosis & $\begin{array}{l}\text { Estimation of overdiagnosis in low-dose CT screening } \\
\text { for lung cancer }\end{array}$ \\
\hline $\begin{array}{l}\text { Disease } \\
\text { definition }\end{array}$ & $\begin{array}{l}\text { Setting thresholds to define the absence or presence } \\
\text { of a disease, or distinguishing between two } \\
\text { subcategories within a disease }\end{array}$ & $\begin{array}{l}\text { Current definitions of airflow obstruction and chronic } \\
\text { obstructive pulmonary disease yield overdiagnosis in } \\
\text { primary care }{ }^{67}\end{array}$ \\
\hline $\begin{array}{l}\text { Incidental } \\
\text { findings }\end{array}$ & $\begin{array}{l}\text { An abnormality found of an unrelated condition during } \\
\text { either diagnostic testing or surgery }\end{array}$ & $\begin{array}{l}\text { Relevance of incidental findings when screening for } \\
\text { a disorder in the abdominal area using multidetector } \\
\text { contrast-enhanced } \mathrm{CT}^{68}\end{array}$ \\
\hline
\end{tabular}

were less common $(3 \%)$. The proportion not related to one particular diagnostic test of interest was $21 \%$. Distributions of diagnostic tests varied significantly depending on the clinical field. Imaging was most prevalent in oncology where it accounted for $48 \%$ of diagnostic tests, mostly related to breast $(53 \%)$ and lung cancer screening $(21 \%)$. Within the field of mental disorders medical examination was often seen in the form of application of the Diagnostic and Statistical Manual of Mental Disorders (DSM) as diagnostic tool. Biomarkers and histology were seen relatively more frequent as diagnostic tests for infectious diseases when compared with other clinical fields.

\section{Screening}

Diagnostic testing was studied in the context of screening in $43 \%$ of studies. There was, however, a skewed distribution between clinical fields. Within oncology, $75 \%$ of all studies were related to screening, whereas for mental disorders, infectious diseases and cardiovascular diseases, this was $15 \%$ or lower.

\section{Overdiagnosis context}

The context in which overdiagnosis was most frequently discussed related to its estimation (53\%). Only within the field of mental disorders was disease definition more frequently discussed than overdiagnosis estimation $(46 \%$ vs $22 \%$ ). Descriptions and example studies on each of the five predefined categories can be found in table 2. The majority of studies discussing overdiagnosis (72\%) were classifiable in one of these categories. Studies that did not fall within any of the five categories were scored in a separate 'other' category (28\%). Results for each of these overdiagnosis contexts are discussed below.

\section{Overdiagnosis estimation}

The most common context of discussion relates to overdiagnosis estimation, accounting for $53 \%$ of all studies. These articles could be divided into two groups. The first were studies attempting to estimate the degree of overdiagnosis in their respective clinical fields (79\%). These often described the impact of a threshold shift or implementation of a diagnostic or screening intervention on the rate of overdiagnosis. Notable examples of this are prostate-specific antigen testing for prostate cancer and mammography for breast cancer. ${ }^{28-33}$ However, several articles estimated overdiagnosis in symptomatic conditions, such as incorrect diagnosis by untrained clinicians in patients presenting with malaria-like symptoms, leading to false-positives and unnecessary treatment. ${ }^{2627}$ This should rather be considered misdiagnosis (incorrect diagnosis of a symptomatic person with a condition they do not have ${ }^{1}$ ) due to inaccuracy of clinical tests used in practice leading to false-positives, incorrect disease labels and overtreatment. The second group represented studies that report methodological approaches for how one should estimate overdiagnosis $(21 \%)$. Differences regarding definitions used, measurement, study design and methods for estimation can lead to different results, ${ }^{34}$ hence there is often a large spread in these estimates, resulting in controversy regarding the true impact of overdiagnosis in the field.

\section{Disease definition}

In $15 \%$ of all studies disease definition was addressed. A relatively high proportion of these studies was addressed in the context of mental disorders $(28 \%)$. Common topics included application of DSM for bipolar disorder, depression and attention deficit hyperactivity disorder, ${ }^{35}$ and physician diagnosis of chronic obstructive pulmonary disease or asthma, which were related to misdiagnosis rather than actual overdiagnosis. ${ }^{37-39}$ The other major contributor was in oncology $(25 \%)$, where the main issue was the transition of benign to malignant growths. Examples of such predisease conditions are ductal carcinoma in situ, early-stage prostate tumours and papillary thyroid carcinoma. $^{40-42}$ 


\section{Overdiagnosis communication}

Communication about overdiagnosis with patients or the public accounted for 3\% of all 1851 publications. This mainly involved the people's understanding of the concept of overdiagnosis and whether they perceived it to be an issue. ${ }^{43-45}$ Other articles dealt with communication of overdiagnosis between the patient and the treating physician, ${ }^{46} 47$ or the development and effectiveness of decision aids. ${ }^{48}$

\section{Other contexts}

Scientific literature on overdiagnosis in genomics and incidental findings were found only sporadically $(0.4 \%$ and $0.8 \%)$. The term overmedicalisation was frequently used in literature to describe medicalisation of normal life events, such as birth, adolescence and death. Quaternary prevention was mostly used to describe the action being taken to prevent overmedicalisation. One of the most commonly observed topics in the other category was drivers and consequences of overdiagnosis. ${ }^{1821} 5051$ These were often mentioned alongside in narrative reviews on overdiagnosis. Furthermore, trend studies were common, describing the possibility of overdiagnosis based on a rapid increase in the number of diagnoses, without any significant decrease in the mortality rate. These studies did not provide an exact overdiagnosis estimate, but rather an indication that overdiagnosis might be occurring or increasing, based on historic data. Another context in which overdiagnosis was commonly addressed, especially in the last couple of years, was its definition. These studies aim at formulating accurate and appropriate definitions of overdiagnosis as well as related terminology (eg, overmedicalisation, overdetection, disease mongering). In addition, some have attempted defining broad overall classifications to provide guidance for distinction between different overdiagnosis subtypes. ${ }^{13} 16$

\section{DISCUSSION}

This scoping review provides insight in the current landscape of overdiagnosis. There is great diversity in study characteristics across medical disciplines and in the contexts in which overdiagnosis is discussed. Some characteristics correlate with specific clinical fields, with, for example, screening occurring predominantly in oncological studies and medical examination being the most prevalently used diagnostic test for mental disorders.

Overdiagnosis is discussed in a variety of contexts; however, three could be distinguished which invoked significant debate: (1) differences in overdiagnosis definition, (2) differences in methods used, leading to varying overdiagnosis estimates and (3) typologies for overdiagnosis.

\section{Overdiagnosis definitions}

The definition of overdiagnosis has been topic of discussion for some time. In a narrow sense, it refers to a diagnosis that does not result in a net benefit for an individual. ${ }^{1}$ This can be viewed within an individual or on a group level, where benefits (early detection of clinically relevant disease) are weighted against the deficits (overdiagnosis and its associated consequences). However, not all included studies give a clear definition, but implicitly use the definition of overdiagnosis as a diagnosis of a 'disease' in an asymptomatic individual, that will never go on to cause symptoms or early death. ${ }^{7}$ This definition is particular to the screening context, but does not apply to a large portion of the studies found in this review that are on testing symptomatic individuals, for example, those with mental disorders. Others have used the relation between pathology and symptoms as a measure of overdiagnosis. ${ }^{5253}$ In the latter, there is no doubt that there is a clear abnormality; however, it is uncertain whether smaller forms of this abnormality still significantly correlate with future clinically relevant disease. Ultimately, the question would be how or even if we should treat these individuals. These examples of definitions demonstrate the heterogeneity and complexity of the concept of overdiagnosis and have led to the discussion regarding the extent or even the existence of overdiagnosis. Which definition researchers use for overdiagnosis needs to be reported completely to be able to judge the applicability of the results.

\section{Methods for overdiagnosis estimation}

Another discussion revolves around variation in estimates of overdiagnosis. Major trials such as the EuropeanRandomized Study of Screening for Prostate Cancer (ERSPC), the National Lung Screening Trial (NLST), the Prostate, Lung, Colorectal and Ovarian (PLCO) Cancer Screening Trial, and the Malmö breast cancer screening trial, often form the basis for these discussions. ${ }^{54-57}$ These trials look into the effects of cancer screening programme. The ERSPC did not provide an overdiagnosis in prostate cancer screening in their initial publication, ${ }^{58}$ but did provide an estimate of $41 \%$ in their 2014 publication. ${ }^{54}$ However, this was obtained through modelling and not calculated directly from the observed data. The NLST merely states that overdiagnosis is presumably not large, as the number of breast cancers diagnosed between the two screening arms is comparable. ${ }^{55}$ And the PLCO and Malmö breast cancer screening trials did not state anything about overdiagnosis. ${ }^{56}{ }^{57}$ The scientific community reacted by using different methods to provide overdiagnosis estimates for these trials. The rate of overdiagnosis that is estimated depends on various features such as the definitions and measurements used, study design and context and estimation approaches applied. ${ }^{12} 34$ 59-63 The latter can be divided in lead time (the time between screening detection and clinical presentation) and excess incidence approach (excess number of cases between a screening and non-screening group), each of which has its merits and issues and requires assumptions to be made. Ultimately, the variety in methodology used has resulted in variation in overdiagnosis estimates and significant controversy between studies. ${ }^{116364}$ 


\section{Overdiagnosis typologies}

Several studies have provided overviews and acknowledged that finding a singular definition of overdiagnosis may not be feasible. However, providing an overdiagnosis classification, aimed at describing subtypes of overdiagnosis, could prove to be useful. Some efforts have been made to create such a typology, however this is challenging as definitions vary widely and classifications can be made over different axes. Hence, this is a complex issue which should be addressed in a systematic manner. A comprehensive typology could aid researchers in their communication as was already suggested in a paper by Moynihan $e t a l^{6}{ }^{6}$ A recent paper by Rogers described the use of maldetection (issues with our understanding of what 'truly' disease is) and misclassification (an implicit or explicit threshold shift resulting in overdiagnosis) ${ }^{13}$ Shortly after this publication, Carter et al described the concepts of predatory, tragic and misdirected overdiagnosis. ${ }^{17}$ Other work by Hofmann takes a more sociological and philosophical point of view. In his 2017 publication, indicative, measurable and observable phenomena are used to describe the different stages in which a phenomenon develops into a clinical manifestation. ${ }^{16}$ In oncology, a tumour-patient classification has been described, relating to tumours that are regressive, non-progressive or truly malignant disease. ${ }^{65}$ Although these works provide great improvement in our understanding of the issues at hand, they do not give further guidance as to how these concepts should be used in clinical research.

\section{Strengths and limitations}

To our knowledge, this is the first scoping review performed on the subject of overdiagnosis. It provides broad insight in the available research on specific topics within overdiagnosis. To appreciate the findings in this review, the following limitations should be considered. First, studies were excluded when they did not have full text available. This may have led to exclusion of a selection of relevant articles, but not a systematic exclusion of a particular range of overdiagnosis studies. The same holds true for the lack of search criteria for iatrogenic disease, overtreatment and overutilisation. The issue in identifying studies discussing overdiagnosis, is that there are no clear selection criteria to find these. Terminologies used to describe overdiagnosis differ between studies, are widely spread and search filters in medical databases are lacking. Hence, our goal was not to perform a comprehensive search. Instead, we aimed at finding a large representative of papers discussing overdiagnosis.

Second, unexpectedly, studies on genomics and incidental findings (or incidentalomas) were largely missed. Forward reference checking revealed that some of the papers not found in our search may use other terminology for describing overdiagnosis, such as the "prevalence of significant findings' or 'diagnostic value'. Using our search strategy, these articles were unfortunately omitted and not included in this review. When researchers are interested particularly in this subset, the information in this review might not suffice.

In summary, overdiagnosis is a topic discussed over medical disciplines, and in a wide array of contexts, from conceptual ideas in definition to practical issues for clinicians in daily practice. The various characteristics of studies looking at overdiagnosis suggest that there may be different (and sometimes multiple) underlying mechanisms through which it may manifest itself. A lack of consensus on what is called overdiagnosis hampers communication between researchers, physicians, patients and policy-makers. The use of overdiagnosis to describe misdiagnosis will dilute its actual meaning, result in linguistic confusion and counterproductive discussion, and should thus be avoided. Providing clarity on the mechanisms that lead to overdiagnosis will aid researchers communicate their results, especially with regard to overdiagnosis estimates. Future methodological studies should focus on establishing a framework to aid clinicians and researchers in understanding the different subtypes of overdiagnosis, their consequences, and provide guidance for selecting appropriate study designs and methods that match the research question of interest.

Contributors KJ, JAHdG and CAN have contributed to the conception and design of the study. KJ, JAHdG and CAN have contributed to the establishment of search and scoring criteria. Article reviewing, scoring and data analysis has been performed by KJ. KJ, JAHdG, JBR, KGMM, LH and CAN have made contributions to the drafting and revising of the article. KJ, JAHdG, JBR, KGMM, LH and CAN have approved the final version to be published and its accuracy and integrity.

Funding Netherlands Organization for Scientific Research (project 918.10.615).

Competing interests None declared.

Provenance and peer review Not commissioned; externally peer reviewed.

Data sharing statement Readers interested in using our database on overdiagnosis for specific purposes related to their respective research are invited to do so by contacting the first author through the corresponding email address.

Open Access This is an Open Access article distributed in accordance with the Creative Commons Attribution Non Commercial (CC BY-NC 4.0) license, which permits others to distribute, remix, adapt, build upon this work non-commercially, and license their derivative works on different terms, provided the original work is properly cited and the use is non-commercial. See: http://creativecommons.org/ licenses/by-nc/4.0/

(c) Article author(s) (or their employer(s) unless otherwise stated in the text of the article) 2017. All rights reserved. No commercial use is permitted unless otherwise expressly granted.

\section{REFERENCES}

1. Carter SM, Rogers W, Heath I, et al. The challenge of overdiagnosis begins with its definition. BMJ 2015;350:h869.

2. ABIM foundation. Choosing wisely around the world. 2015. http:// www.choosingwisely.org/resources/updates-from-the-field/choosingwisely-around-the-world/ (accessed 4 Jan 2017).

3. Otte JA. Less is more medicine. http://www.lessismoremedicine. com/projects/ (accessed 9 May 2017).

4. Welch GH. Overdiagnosed: Making People Sick in the Pursuit of Health, 2010.

5. Black WC. Overdiagnosis: an underrecognized cause of confusion and harm in cancer screening. J Natl Cancer Inst 2000;92:1280-2.

6. Moynihan R, Doust J, Henry D. Preventing overdiagnosis: how to stop harming the healthy. BMJ 2012;344:e3502. 
7. Welch HG, Black WC. Overdiagnosis in cancer. $J$ Natl Cancer Inst 2010;102:605-13.

8. Etzioni R, Penson DF, Legler JM, et al. Overdiagnosis due to prostate-specific antigen screening: lessons from U.S. prostate cancer incidence trends. J Natl Cancer Inst 2002;94:981-90.

9. Pohl H, Welch HG. The role of overdiagnosis and reclassification in the marked increase of esophageal adenocarcinoma incidence. J Natl Cancer Inst 2005;97:142-6.

10. Preventing Overdiagnosis. Preventing overdiagnosis conference. http://www.preventingoverdiagnosis.net/ (accessed 4 Jan 2014).

11. Bae JM. Overdiagnosis: epidemiologic concepts and estimation. Epidemiol Health 2015;37:e2015004.

12. Bach PB. Overdiagnosis in lung cancer: different perspectives, definitions, implications. Thorax 2008;63:298-300.

13. Rogers WA, Mintzker Y. Getting clearer on overdiagnosis. J Eval Clin Pract 2016;22:580-7.

14. Hofmann BM. Conceptual overdiagnosis. a comment on wendy rogers and yishai mintzker's article "getting clearer on overdiagnosis". J Eval Clin Pract 2017;23.

15. Rogers WA, Mintzker Y. Response to bjorn hofmann: Clarifying overdiagnosis without losing conceptual complexity. J Eval Clin Pract 2017;23.

16. Hofmann B. Defining and evaluating overdiagnosis. J Med Ethics 2016:715-6.

17. Carter SM, Degeling C, Doust J, et al. A definition and ethical evaluation of overdiagnosis. J Med Ethics 2016:705-14.

18. Paris J, Bhat V, Thombs B. Is adult attention-deficit hyperactivity disorder being overdiagnosed? Can J Psychiatry 2015;60:324-8.

19. Pathirana T, Clark J, Moynihan R. Mapping the drivers of overdiagnosis to potential solutions. BMJ 2017;358:j3879.

20. Hofmann BM. Too much technology. BMJ 2015;350:h705.

21. Doust J, Glasziou P. Is the problem that everything is a diagnosis? Aust Fam Physician 2013;42:856-9.

22. Winters BS, Solarz M, Jacovides CL, et al. Overdiagnosis of pulmonary embolism: evaluation of a hypoxia algorithm designed to avoid this catastrophic problem. Clin Orthop Relat Res 2012;470:497-502.

23. Suh JM, Cronan JJ, Healey TT. Dots are not clots: the over-diagnosis and over-treatment of PE. Emerg Radiol 2010;17:347-52.

24. Bruchmüller K, Margraf J, Schneider S. Is ADHD diagnosed in accord with diagnostic criteria? Overdiagnosis and influence of client gender on diagnosis. J Consult Clin Psychol 2012;80:128-38.

25. Bonati M, Reale L. Reducing overdiagnosis and disease mongering in ADHD in Lombardy. BMJ 2013;347:f7474.

26. Harchut K, Standley C, Dobson A, et al. Over-diagnosis of malaria by microscopy in the kilombero valley, Southern Tanzania: an evaluation of the utility and cost-effectiveness of rapid diagnostic tests. Malar $J$ 2013;12:159.

27. Mwanziva C, Shekalaghe S, Ndaro A, et al. Overuse of artemisinincombination therapy in mto wa mbu (river of mosquitoes), an area misinterpreted as high endemic for malaria. Malar J 2008;7:232

28. Pelzer AE, Colleselli D, Bektic J, et al. Over-diagnosis and underdiagnosis of screen- vs non-screen-detected prostate cancers with in men with prostate-specific antigen levels of $2.0-10.0 \mathrm{ng} / \mathrm{mL}$. BJU Int 2008;101:1223-6.

29. Heijnsdijk EA, de Carvalho TM, Auvinen A, et al. Cost-effectiveness of prostate cancer screening: a simulation study based on ERSPC data. J Natl Cancer Inst 2015;107:366.

30. Arnsrud Godtman R, Holmberg E, Lilja H, et al. Opportunistic testing versus organized prostate-specific antigen screening: outcome after 18 years in the Göteborg randomized population-based prostate cancer screening trial. Eur Urol 2015:68:354-60.

31. Beckmann K, Duffy SW, Lynch J, et al. Estimates of over-diagnosis of breast cancer due to population-based mammography screening in South Australia after adjustment for lead time effects. J Med Screen 2015;22:127-35.

32. Seigneurin $\mathrm{A}$, Labarère $\mathrm{J}$, François $\mathrm{O}$, et al. Overdiagnosis and overtreatment associated with breast cancer mammography screening: a simulation study with calibration to population-based data. Breast 2016;28:60-6.

33. Gunsoy NB, Garcia-Closas M, Moss SM. Estimating breast cancer mortality reduction and overdiagnosis due to screening for different strategies in the United Kingdom. Br J Cancer 2014;110:2412-9.

34. Etzioni R, Gulati R, Mallinger L, et al. Influence of study features and methods on overdiagnosis estimates in breast and prostate cancer screening. Ann Intern Med 2013;158:831-8.

35. Phelps J, Ghaemi SN. The mistaken claim of bipolar 'overdiagnosis': solving the false positives problem for DSM-5/ICD-11. Acta Psychiat Scand 2012;126:395-401.

36. Sciutto MJ, Eisenberg M. Evaluating the evidence for and against the overdiagnosis of ADHD. J Atten Disord 2007;11:106-13.
37. García-Rio F, Soriano JB, Miravitlles M, et al. Overdiagnosing subjects with COPD using the 0.7 fixed ratio: correlation with a poor health-related quality of life. Chest 2011;139:1072-80.

38. Güder G, Brenner S, Angermann CE, et al. "GOLD or lower limit of normal definition? A comparison with expert-based diagnosis of chronic obstructive pulmonary disease in a prospective cohortstudy". Respir Res 2012;13:13.

39. Aaron SD, Vandemheen KL, Boulet LP, et al. Overdiagnosis of asthma in obese and nonobese adults. CMAJ 2008;179:1121-31.

40. Evans AJ, Pinder SE, Ellis IO, et al. Screen detected ductal carcinoma in situ (DCIS): overdiagnosis or an obligate precursor of invasive disease? J Med Screen 2001:8:149-51.

41. Van der Kwast TH, Roobol MJ. Defining the threshold for significant versus insignificant prostate cancer. Nat Rev Urol 2013;10:473-82.

42. Vaccarella S, Dal Maso L, Laversanne M, et al. The impact of diagnostic changes on the rise in thyroid cancer incidence: a population-based study in selected high-resource countries. Thyroid 2015;25:1127-36.

43. Moynihan R, Nickel B, Hersch J, et al. What do you think overdiagnosis means? A qualitative analysis of responses from a national community survey of Australians. BMJ Open 2015;5:e007436

44. Hersch J, Jansen J, Barratt A, et al. Women's views on overdiagnosis in breast cancer screening: a qualitative study. BMJ 2013;346:f158.

45. Moynihan R, Nickel B, Hersch J, et al. Public opinions about overdiagnosis: a national community survey. PLoS One 2015;10:e0125165.

46. van Agt H, Fracheboud J, van der Steen A, et al. Do women make an informed choice about participating in breast cancer screening? A survey among women invited for a first mammography screening examination. Patient Educ Couns 2012;89:353-9.

47. Wegwarth O, Gigerenzer G. Less is more: Overdiagnosis and overtreatment: evaluation of what physicians tell their patients about screening harms. JAMA Intern Med 2013;173:2086-7.

48. Bae JM. Development and application of patient decision aids. Epidemiol Health 2015;37:e2015018.

49. Hersch J, Barratt A, Jansen J, et al. Use of a decision aid including information on overdetection to support informed choice about breast cancer screening: a randomised controlled trial. Lancet 2015:385:1642-52.

50. Day M. Drug industry is partly to blame for overdiagnosis of bipolar disorder, researchers claim. BMJ 2008;336:1092-3.

51. Carneiro AV. Screening for coronary artery disease in assymptomatic adults is not recommended, so why is it still done? Rev Port Cardiol 2004;23:1633-8.

52. Hoffman JR, Carpenter CR. Guarding against overtesting, overdiagnosis, and overtreatment of older adults: thinking beyond imaging and injuries to weigh harms and benefits. J Am Geriatr Soc 2017;65:903-5

53. de Roos MA, van der Vegt $\mathrm{B}$, de Vries J, et al. Pathological and biological differences between screen-detected and interval ductal carcinoma in situ of the breast. Ann Surg Oncol 2007;14:2097-104.

54. Schröder FH, Hugosson J, Roobol MJ, et al. Screening and prostate cancer mortality: results of the European Randomised Study of Screening for Prostate Cancer (ERSPC) at 13 years of follow-up. Lancet 2014;384:2027-35.

55. Aberle DR, Adams AM, Berg CD, et al. Reduced lung-cancer mortality with low-dose computed tomographic screening. $N$ Engl J Med 2011;365:395-409.

56. Andriole GL, Crawford ED, Grubb RL, et al. Prostate cancer screening in the randomized prostate, lung, colorectal, and ovarian cancer screening trial: mortality results after 13 years of follow-up. J Natl Cancer Inst 2012;104:125-32.

57. Andersson I, Aspegren K, Janzon L, et al. Mammographic screening and mortality from breast cancer: the Malmö mammographic screening trial. BMJ 1988;297:943-8.

58. Schröder FH, Hugosson J, Roobol MJ, et al. Screening and prostatecancer mortality in a randomized European study. N Engl $\mathrm{J}$ Med 2009;360:1320-8.

59. Wu D, Pérez A. A limited review of over diagnosis methods and long term effects in breast cancer screening. Oncol Rev 2011;5:143-7.

60. Duffy SW, Lynge $\mathrm{E}$, Jonsson $\mathrm{H}$, et al. Complexities in the estimation of overdiagnosis in breast cancer screening. Br J Cancer 2008;99:1176-8.

61. de Gelder R, Heijnsdijk EA, van Ravesteyn NT, et al. Interpreting overdiagnosis estimates in population-based mammography screening. Epidemiol Rev 2011;33:111-21.

62. Draisma G, Etzioni R, Tsodikov A, et al. Lead time and overdiagnosis in prostate-specific antigen screening: importance of methods and context. J Natl Cancer Inst 2009;101:374-83. 
63. Puliti D, Miccinesi G, Paci E. Overdiagnosis in breast cancer: design and methods of estimation in observational studies. Prev Med 2011;53:131-3.

64. Davidov O, Zelen M. Overdiagnosis in early detection programs. Biostatistics 2004;5:603-13.

65. Marcus PM, Prorok PC, Miller AB, et al. Conceptualizing overdiagnosis in cancer screening. J Natl Cancer Inst 2015;107:djv014.

66. Patz EF, Pinsky P, Gatsonis C, et al. Overdiagnosis in low-dose computed tomography screening for lung cancer. JAMA Intern Med 2014;174:269-74.
67. Schermer TR, Smeele IJ, Thoonen BP, et al. Current clinical guideline definitions of airflow obstruction and COPD overdiagnosis in primary care. Eur Respir J 2008;32:945-52.

68. Sconfienza LM, Mauri G, Muzzupappa C, et al. Relevant incidental findings at abdominal multi-detector contrast-enhanced computed tomography: a collateral screening? World J Radiol 2015;7:350-6.

69. Hall AE, Chowdhury S, Pashayan N, et al. What ethical and legal principles should guide the genotyping of children as part of a personalised screening programme for common cancer? J Med Ethics 2014;40:163-7. 